\title{
Vibrations des structures en forme de plaques et de coques en présence d'un milieu fluide incompressible
}

\section{Vibration of plate and shell structures in an incompressible fluid medium}

Séverin Casacci

Directeur technique

Société Neyrpic - Grenoble
Gilbert Caillot

Ingénieur

Société Neyrpic - Grenoble
René-Jean Gibert

Chef du Laboratoire des Études de Vibrations

C.E.A. Saclay
Nous utiliserons dans la suite, les notations genérales suivantes :

$E$, $V$ et $P$ respectivement module de Young, coefficient de Poisson et masse volumique du matériau de la structure immergée,

$\rho_{L}$ masse volumique du milieu fluide baignant la structure,

- potentiel des vitesses du mouvement de fluide induit par les vibrations de la structure.

\section{VIBRATIONS DE PLAOUES DANS UN MILIEU CONFINE}

\subsection{Bande rectangulaire indéformable = exemple de masse ajoutée}

On considère une bande indéformable de largeur unitê et de longueur 2 , pouvant pivoter autour ' 'un axe passant par le milieu de ce côté. etce bate d'un fluide incompressible est limité par un fond fixe distant de $L$ de l'axe de la paroi pivotante.

Au cours du mouvement, la position d'un point d'abscisse $x$ de la bande est donné par sa cote y :

$$
\begin{array}{cc}
y-a\left(x-\frac{\ell}{2}\right) \text { Ein we } & \begin{array}{c}
\text { (Axe de rotation au point } \\
x=2 / 2 \\
y=0)
\end{array} \\
\text { soit } y=-\frac{4 a}{l} \sum_{n=0}^{\infty}\left(\frac{\ell}{(2 n+1) \pi}\right)^{2} \cos (2 n+1) \frac{\pi x}{\ell}
\end{array}
$$

En se linitant au domaine des petits mouvements, le potentiel de l'écoulement induit par les déplacements de la plaque doit satisfaize à

'équation $\Delta s=0$ et aux conditions aux limites:

$$
\begin{aligned}
& \frac{\partial \phi}{\partial x}=0 \quad \text { pour } x=0 \text { et } x=l \\
& \frac{\partial \phi}{\partial y}=0 \quad \text { pour } y=L \\
& \frac{\partial \phi}{\partial y}=\frac{\partial y}{\partial t} \text { pour } y=0
\end{aligned}
$$

Ce pocentiel peut donc ce représenter par le dêveloppement en sêrie

$$
n=\omega \cos \omega t \sum_{n=0}^{\infty} \gamma_{n} \cdot \frac{\operatorname{ch} \frac{(2 n+1)}{\ell} \pi(y-L)}{\sin (2 n+1) \cdot \frac{\pi L}{\ell}} \cdot \cos (2 n+1) \frac{\pi x}{l}
$$

qui vérifie directement l'équation de Laplace et les conditions (1) et (2). La condition (3) permet de calculer les coefficients $Y_{n}$ par identification et d'obtenir :

$$
\gamma_{n}=\frac{4 a \ell^{2}}{\left((2 n+1)[]^{3}\right.}
$$

Considérons le potentiel des vitesses pour une vitesse angulaire de rotation Considérons le por
unité, ił vient

$$
\left[\frac{\partial \phi}{\partial y}\right]_{y=0}=\left(\frac{\partial y}{\partial t}\right)_{y=0} \#\left(x-\frac{\ell}{2}\right)
$$

et dans ces conditions :

$$
\phi=4 \ell^{2} \sum_{n=0}^{\infty} \frac{1}{((2 n+1) \pi)^{2}} \cdot \frac{1}{\operatorname{th}(2 n+1) \frac{n L}{l}} \cdot \cos \frac{(2 n+1)}{l} \pi x
$$

L'inertie ajoutée par unitế de largeur est donnée par l'intếgrale :

$$
I=-\rho_{L} \int_{c} \phi \cdot \frac{\partial \phi}{\partial n} \cdot d s
$$

1e domaine d'intégration s'étendant à toutes les surfaces frontières. Compte tenu des conditions aux limites, seule la bande elle-même subsiste corme domaine d'intégration.

L'inertie ajoutêe pour une bande de lägeur unitê, calculêe dans ces conditions est égale à :

$$
I=\frac{8 \rho_{L} 2^{4}}{\pi^{5}} \sum_{n=0}^{\infty} \frac{1}{(2 n+1)^{5}} \cdot \frac{1}{\operatorname{th}(2 n+1) \frac{\pi L}{8}}
$$

On peut remarquer que lorsque le fond du canal est rejetê à $l^{\prime}$ infini l'inertie ajoutée par unité de largeur tend vers la valeur:

$$
I \simeq \frac{8 p_{L} 2^{4}}{\pi^{5}}
$$

L'évolution de l'inertie ajoutếe en fonction de l'êloignement relatif $\frac{L}{2}$ du fond du conduit est représentée sur la figure 1 . On constate que l'effet de confinement diminue rapidement avec les valeurs de l'êloignement relatif; il est inférieur à $1 \%$ dès $\frac{L}{2}=0,9$. Ceci est dû au fait que c'est le liquide proche de la paroi mobile qui constitue 1 'apport le plus important du point les mouvements des différentes tranches de fluide au cours de la vibration: le fluide considéré étant incompressible, les mouvements sont en phase.

LA HOUILLE BLANCHE/N $1 / 2-1980$ 


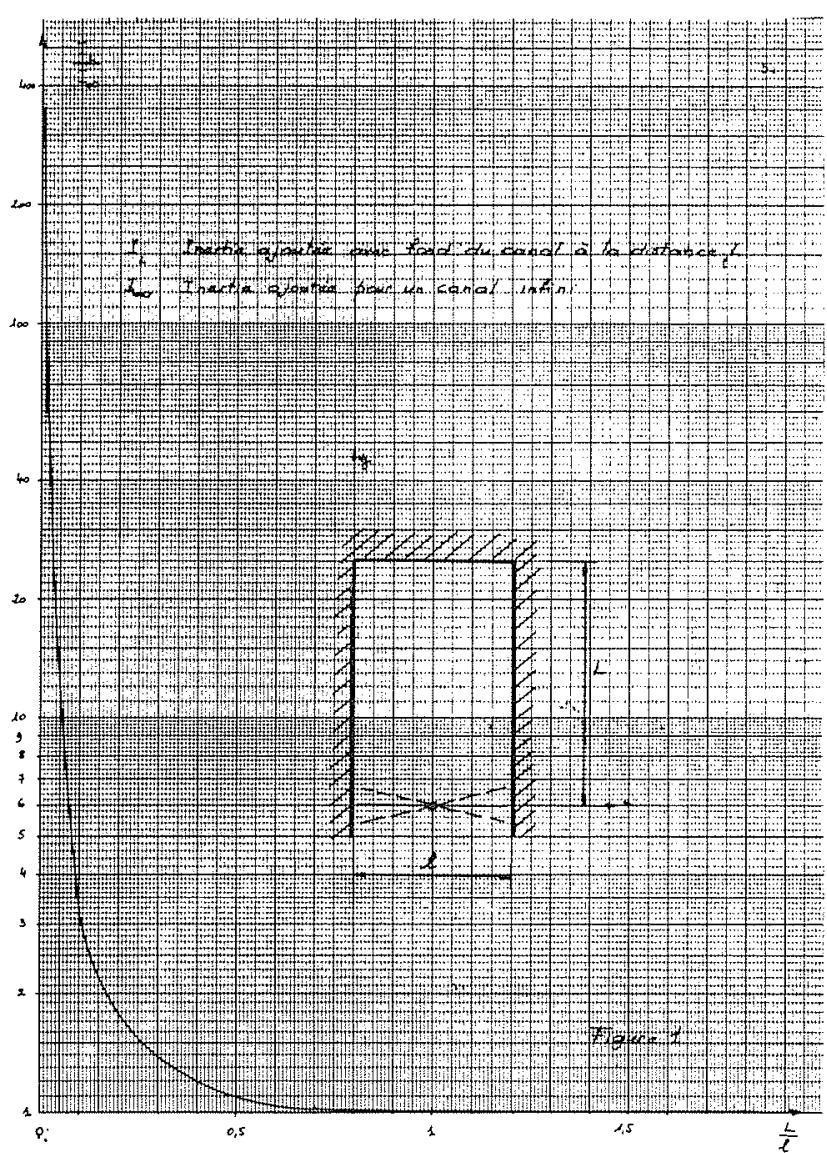

Figure 1

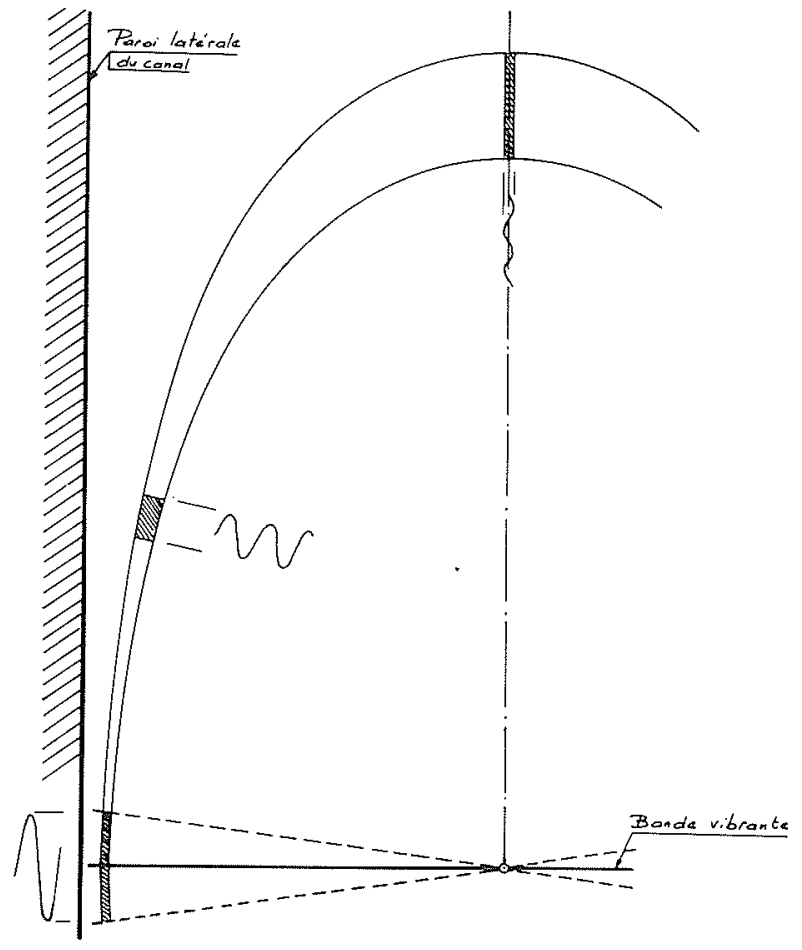

$$
\begin{aligned}
& \text { Amplitude comparaiz an diffirants points, du fluide } \\
& \text { mis an mouvanent par la bande vibrante. }
\end{aligned}
$$

Figure 2

\subsection{Bande rectangulaire encastrée}

On considère une bande de largeur unité encastrếe à ses deux extrếmitếs dan les parois parallèles d'un canal rempli de fluide incompressible.

La plaque est baigneae sur ses deux faces. On désigne par :

a et e respectivement longueur et épaisseur de la plaque

$k^{2}=12\left(\frac{a}{e}\right)^{2} \quad ; \quad D=\frac{E e^{3}}{12\left(1-v^{2}\right)}$

$x=$ axe longitudinal de la bande, variable adimensionnelle $x=\frac{x}{a}$

$z$ = axe perpendiculaire à la plaque, variable adinensionnelle $z=\frac{z}{3}$

Cet axe définit un côté positif et un côté négatif de la plaque

$w=$ déplacement selon I'axe des $z$, variable adimensionnelle $w=\frac{w}{a}$

$\dot{\omega}=$ vitesse selon la normale à la plaque

$q=$ effort tranchant dans $l a$ bande; variable adimensionnelle $Q=\frac{q a^{2}}{D}$

$\mathrm{m}$ - moment de Elexion dans la bande; variable adimensionnelle $M=\frac{\mathrm{ma}}{\mathrm{D}}$

f effort extêrieur normal à la plaque

Les équations d'équilibre d'un élément de plaque s'écrivent :

$$
\begin{aligned}
& \frac{\partial q}{\partial x}+f=0 \\
& \frac{\partial m}{\partial x}+q=0 \\
& \frac{m}{D}=\frac{\partial \omega^{2}}{\partial x^{2}}=\frac{\partial y}{\partial x} \quad \text { en posant } y=\frac{\partial w}{\partial x}
\end{aligned}
$$

Le moment a s'exprime par

Les équations (1) et (2) deviennent alors:

$$
\begin{aligned}
& \frac{\partial^{2} Q}{\partial x^{2}}=-\frac{a^{3}}{D} \frac{\partial f}{\partial x} \\
& \frac{\partial^{2} y}{\partial x^{2}}=-Q
\end{aligned}
$$

L'effort extêrieur normal à la plaque correspond à l'action de la pression p exercée par le fluide et aux effets d'inertie sur la plaque.

$$
f=-p-\rho e \ddot{w}
$$

Les potentiels des écoulements induits de part et d'autre du panneau ( $\phi$ du côté des $z$ positifs, $\$$ du côté des $z$ négatifs) doivent satisfaire à l'équation de Laplace ef aux conditions aux limites suivantes :

$$
\begin{aligned}
& \frac{\partial \phi_{+}}{\partial z}=\frac{\partial \Phi_{-}}{\partial z}=a \dot{w} \\
& \frac{\partial \Phi_{+}}{\partial x}=\frac{\partial \Phi_{-}}{\partial X}=0 \text { pour } x=0 \text { et } x=1 \\
& \frac{\partial \Phi_{+}}{\partial z}=0 \text { pour } z=+\infty \text { et } \frac{\partial \Phi_{-}}{\partial z}=0 \text { pour } z=-\infty \text { (6) }
\end{aligned}
$$

Le đéplacement d'un point du panneau peut se représenter par le dêveloppement

$$
W=\frac{w}{a}=\cos B+\sum_{n=1}^{\infty} w_{n} \cos n \pi x
$$

Si on étudie les dêformations antisymétriques du panneau, c'est-à-dire celles quí n'entraînent pas de variation de volume du fluide supposé incompressible, le developpement ne comporte que des termes impairs.

$$
w=\cos B t \sum_{n=1,3,5}^{\infty} w_{n} \cos \alpha_{n} x \text { en posant } \alpha_{n}=n f l
$$

relation qui satisfait la condition de pente nulle à l'encastrement. La vitesse de déplacement d'un point du panneau s'exprime par :

$$
\dot{w}=a \dot{w}=-a B \sin B t \sum_{n=1,3,5}^{\infty} w_{n} \cos \alpha_{n} x
$$


Les potentiels des écoulements peuvent se représenter par les développements suivants:

- du côté des z positifs :

$$
\Phi_{+}=a^{2} \beta \sin B t \sum_{n=1,3,5}^{\infty} \frac{w_{n}}{\alpha_{n}} \cdot \cos \alpha_{n} x \cdot e^{-\alpha_{n} z}
$$

- du côtê des z nêgatifs :

$$
\phi_{-}=-a^{2} \beta \sin \beta t \sum_{n=1,3,5}^{\infty} \frac{w_{n}}{\alpha_{n}} \cdot \cos \alpha_{n} x \cdot e^{\alpha_{n} z}
$$

Ces relations satisfont à l'êquation de Laplace et aux conditions (6), (7) et (8).

en posant $K=\frac{P_{L}{ }^{a}}{C_{e}}$

L'êquation (3) devient alors :

$$
\begin{aligned}
& \frac{\partial^{2} Q}{\partial x^{2}}=k^{2} \quad \gamma^{2} \cos B t \sum_{n=1,3,5}^{\infty}\left(\alpha_{n}+2 k\right) w_{n} \sin \alpha_{n} x \\
& \text { avec }: \quad: \quad \gamma^{2}=0 \frac{\left(1-v^{2}\right) a^{2} B^{2}}{E}
\end{aligned}
$$

Si on désigne par $Q(0)$ et $Q(1)$ les cisaillements en $x=0$ et $x=1$, ils sont tels que $Q(0)=Q(1)$.

Envisageons le changement de variable:

$$
\begin{aligned}
& Q=Q^{*}+Q(0) \\
& Q^{*} \begin{array}{l}
\text { devra satisfaire aux conditions } Q^{*}=0 \text { pour } X=0 \text { er } X=1 \\
\text { et par suite être dêveloppé en sêrie de } \sin \alpha_{n} X
\end{array}
\end{aligned}
$$

Q peut donc se représenter sous la forme

$$
Q=\left(\sum_{n=1,3,5}^{\infty} Q_{n}^{*} \sin \alpha_{n} x+4 Q_{(0)} \sum_{n=1,3,5}^{\infty} \frac{\sin \alpha_{n} x}{\alpha_{n}}\right) \cos B t
$$

Le système (9) et (4) se transforme par identification terme à terme en un systène algébrique :

$$
\left\{\begin{aligned}
-\alpha_{n}^{2} Q^{x} & =k^{2} \gamma^{2}\left(\alpha_{n}+2 K\right) W_{n} \\
\alpha_{n}^{3} W_{n} & =-Q^{*}-4 \frac{Q(0)}{\alpha_{n}}
\end{aligned}\right.
$$

La rêsolution de ce système conduit à :

$$
w_{n}=\frac{-4 a_{n} Q(0)}{\frac{a^{5}-k^{2} \gamma^{2}\left(a_{n}+2 k\right)}{n}}
$$

et la condition de déformation nulle au niveau de l'encastrement $\left.{ }^{(W}(0)=W_{(1)}=0\right)$ sera faite si:

$$
\sum_{n=1,3,5}^{\infty} \frac{a_{n}}{\alpha_{n}^{5}-k^{2} \gamma^{2}\left(a_{n}+2 k\right)}=0
$$

Les valeurs de $\gamma$ perwettant de satisfaire l'équation (12) conduisent aux valeurs des fréquences propres.

Les termes de la série diminuent très rapidement lorsque l'ordre de $n$ s'élève. Pour calculer la première fréquance propre il suffit de considêrer les deux ou trois fremiers termes et de remplacer les autres par $\frac{1}{\alpha^{4}}$ Ces derniers se calculent aisément si on tient compte de la relation :

$$
\sum_{n=1}^{\infty} \frac{1}{a^{4}}=\frac{1}{96}
$$

Remarquons que dans le vide, les fréquences propres seraient données par les valeurs de $\gamma$ satisfaisant $a$

$$
\sum_{n=1,3,5}^{\infty} \frac{1}{\alpha_{n}^{4}-k^{2} \gamma^{2}}=0
$$

On constate ainsi que les frêquences propres sont considérablement affectées par la présence de fluide. Ainsi par exemple, une bande d'acier dont le rapport de la longueur à l'épaisseur serait égal a 100 , a une fréquence propre 2,6 to
est vide.

\subsection{Plaque rectangulaire encastrée sur ses 4 côtés}

Soit une plaque obstruant un canal de section carrée, d'axe pexpendiculaire au plan de la plaque.

On considère un systême orthonorté $x_{1} x_{2} x_{3}$; le plan de la plaque étant défini par $x_{1}, x_{2}$.

On désigne par :

a et e respectivenent la longueur du côtê et l'épaisseur de la plaque :

$$
\begin{aligned}
& k^{2}=12 \frac{a^{2}}{e^{2}}, D=\frac{E e^{3}}{12(1-v)} \\
& B=1 a \text { pulsation de la vibration }\left(\gamma^{2}=\frac{e\left(1-v^{2}\right) a^{2} B^{2}}{E} \text { pulsation réduite }\right) \\
& k=\frac{D_{L}^{2}}{D^{2}} \\
& a_{i}=i \frac{1}{1} \\
& B_{i j}^{2}=\alpha_{i}^{2}+a_{j}^{2}
\end{aligned}
$$

Une étude analogue à celle effectuêe en 1.2 conduit à un système infini d'équations qui $n^{\prime}$ admet une solution que si son dététminant caractériscique s'annule:

$$
\left\|a_{i j}\right\|=0
$$

où $\quad a_{i j}=D_{i j}^{-1}+\delta_{i j} \sum_{1=1,3, \ldots} D_{i 1}^{-1}$

Dũ $\quad D_{i j}=k^{2} \gamma^{2}\left(k_{i j}+1\right)-\beta_{i j}^{4}$

Ce dêterminant dêveloppể s'êcrit :

\begin{tabular}{|c|c|c|c|}
\hline$D_{11}^{-1}+\sum_{n=1} D_{1 \mathrm{n}}^{-1}$ & $D_{13}^{-1}$ & $D_{15}^{-1}$ & $D_{17}^{-1}$ \\
\hline$D_{31}^{-1}$ & $D_{33}^{-1}+\sum_{n=1} D_{3 n}^{-1}$ & $D_{35}^{-1}$ & $D_{37}^{-1}$ \\
\hline$D_{51}^{-1}$ & $D_{53}^{-1}$ & $D_{55}^{-1}+\sum_{n=1} D_{5 n}^{-1}$ & $D_{57}^{-1}$ \\
\hline$D_{71}^{-1}$ & $D_{73}^{-1}$ & $D_{75}^{-1}$ & $D_{77}^{-1}+\sum_{n=1} D_{7 n}^{-1}$ \\
\hline
\end{tabular}

La première fréquence propre correspond approximativement à la valeur de $Y_{1}$ qui annule l'équation:

$$
\begin{aligned}
& D_{13}=k^{2} \gamma_{1}^{2}\left(K_{13}+1\right)-B_{13}^{4}=0 \\
& K_{13}=\frac{\rho_{L}^{3}}{\rho e} \quad B_{13}^{-1}
\end{aligned}
$$

avec :

et :

$$
B_{13}^{2}=\pi^{2}\left(1+3^{2}\right)=10 \pi^{2}
$$

đ'où la première ftếquence propre pour un panneau carré obturant un conduit de même section rempli d'un fluide incompressible.

$$
f_{1}=\frac{10 \pi}{4 \sqrt{3\left(1-v^{2}\right)\left(k_{13}+1\right)}} \frac{e}{a^{2}} \sqrt{\frac{E}{\rho}}
$$

Remarquons que si le conduit supposé infini existe aussi du côtê des $z$ négatifs, il faut remplacer le $\mathrm{K}_{13}$ deffini par (4) par $2 \mathrm{~K}_{13}$. 


\section{VIBRATIONS DE COQUES}

\section{Coques cylindriques}

On considère deux coques de longueur infinie, d'axes parallèles et excentrếs de la distance $e$. Un fluide incompressible et non visqueux remplit 'intervalle entre les deux coques dont les rayons respectifs sont $R$ (coque extérieure) et $r$ (coque intérieure)

Pour faciliter 1'expression du potentiel, on utilise un système de coordonnées bipolaires.

Dans le plan orthonorné $x, y$, considérons le systèrne de coordonnées orthogonales et tel que

$$
z=\alpha+i \beta=\log \frac{z+i b}{z-i b}=\frac{x+i(y+b)}{x+i(y-b)}
$$

où : $z=x+$ iy

$$
z \bar{z}=p^{2}
$$

soit : $\quad e^{z}=\frac{p^{2}-b^{2}+2 i x b}{p^{2}+b^{2}-2 b y}$

Expression qui peut se mettre sous la forme :

$$
e^{\alpha}(\cos \beta+i \sin \beta)=\frac{\left(\left(p^{2}+b^{2}\right)^{2}-4 y^{2} b\right)^{1 / 2}}{p^{2}+b^{2}-2 y b}(\cos \beta+i \sin \beta)
$$

d'où $l^{\prime}$ on peut tirer en posant $h=\frac{2 b}{\left(\left(\rho^{2}-b^{2}\right)^{2}+4 x^{2} b^{2}\right)^{1 / 2}}$

$$
\begin{aligned}
& h x=\sin \beta \\
& \cos \beta=\left(\rho^{2}-b^{2}\right)\left(\left(\rho^{2}-b^{2}\right)^{2}+4 x^{2} b^{2}\right)-1 / 2 \\
& e^{2 a}=\frac{\rho^{2}+b^{2}+2 y b}{\rho^{2}+b^{2}-2 y b}
\end{aligned}
$$

Après quelques transformations simples, il vient :

$$
\begin{aligned}
& e^{a}=\left(1+y^{2} h^{2}\right)^{1 / 2}+y h \text { et } \\
& \text { yh }=\operatorname{sha}
\end{aligned}
$$

Corne :

$$
h^{2} x^{2}+h^{2} y^{2}=h^{2} p^{2}=\sin ^{2} B+\operatorname{sh}^{2} \alpha=\operatorname{ch}^{2} \alpha-\cos ^{2} \beta
$$

On obtient :

$$
h b=\operatorname{ch} \alpha-\cos \beta
$$

Les coorodonnées aet $z$ sont fonc reliées aux päramètres b, $x$ ez $y$ par les relations (1), (2) et (3).

Forme des courbes correspondant à upuis 3 constancs (voir figure 3)

\section{Courbes $a=\alpha_{i}=c c e$}

Par élimination de bet hentre (1), (2), et (3), on obtient la relation

$$
x^{2}=\left(y-b c t h \alpha_{i}\right)^{2}=\frac{b^{2}}{\operatorname{sh}^{2} \alpha_{i}}
$$

equation d'un cercle de rayon $r_{i}=\frac{b}{\operatorname{stu}_{i}}$ et centre sur l'axe des $y$ a

$d_{i}=r_{i}$ ch $\alpha_{i}$, c'est-ì-dire que pour tous les $a_{i} \neq 0 d_{i}>r_{i}$ (cercles non sécants avec 1 'axe des $x$ )

Les cas Limites donnent:

$$
\begin{aligned}
& a=0=\text { cercle confondu avec } l^{\prime} \text { axe des } x \\
& a=\infty=\text { cercle confondu avec le point } x=0, y=b
\end{aligned}
$$

\section{Courbes $B=B_{i}=$ cte}

De façon analogue, on obtient l'équation $d^{\prime}$ un cercle de rayon $\frac{b}{\sin B}$. et dont le centre est situé sur $l$ 'axe des $x$ à une distance $b \operatorname{cotg} B_{1}$ de celúi des $y$.

Ces cercles passenc tous par les points $(x=0, y=+b)$ et sont orthozonaux au réseau $a_{i}=$ cte.

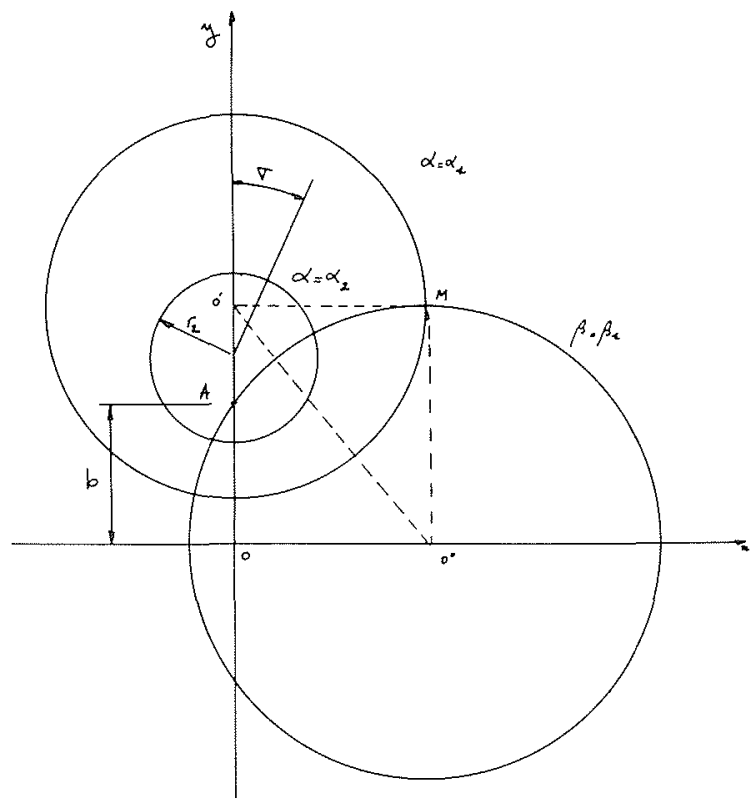

Figure 3

Envisageons le cas qu'une coque située à l'intérieur d'une enceinte cylindriqu indëformable.

Pour étudier la masse ajoutée du fluide incompressible et non visqueux, compris entre la coque et l'enceinte cylindriques dont les surfaces moyennes admettent pour sections respectivement les cercles $\alpha=\alpha_{2}$ et $\alpha=\alpha_{1}$ avec $\alpha_{1}, \alpha_{2}$ constants,

$$
\psi=b v(t) \sum_{n=1,2}^{\infty} \frac{1}{n} \frac{\operatorname{chn}\left(\alpha-\alpha_{1}\right)}{\operatorname{shn}\left(\alpha_{2}-\alpha_{1}\right)}\left(a_{n} \cos n \&+b_{n} \sin n \beta\right)
$$

La vitesse u, dans le sens desa positifs s'exprime par :

$$
u_{\alpha}=h \frac{\partial \phi}{\partial \alpha}=(\operatorname{ch} \alpha-\cos \beta) v_{(t)} \quad \sum \frac{\operatorname{shn}\left(\alpha-\alpha_{1}\right)}{\operatorname{shn}\left(\alpha_{2}-\alpha_{1}\right)}\left(a_{n} \cos n \beta+b_{n} \sin n \beta\right)
$$

Cetre expression montre que $u_{\alpha} s^{\prime}$ annule sur le cercle $a=\alpha_{1}$ (parois rigides de l'enceinte) et done que le champ des vitesses dêfini par le potentiel choisi satisfait bien aux conditions aux limites a ce niveau.

Au niveau de la coque $a_{2}$ la vitesse est donnée par :

$$
u_{a_{2}}=\left(c h a_{2}-\cos \beta\right) v_{(c)} \sum_{n=1,2}\left(a_{n} \cos n \hat{b}+b_{n} \sin n B\right)
$$

Masse duntúe

La masse ajoutée par unité de longueur est donnée par l'intúgrale :

$$
m^{\prime}=\rho_{L} / \int_{\Sigma} \cdot \frac{\partial p}{\partial n} d S
$$

le domaine d'intégration s'étendant à toutes les surfaces frontières.

2.1. Coque indeformable

Envisageons le cas d'une cogue se deplaçant en bloc dans la direction $a=\sigma_{0}$. Dans ces conditions 1 a coque $\alpha_{2}$ est sourise a un nouvemene tel que

$$
u_{a_{2}}=v_{(r)} \cos \left(\sigma-\sigma_{0}\right)=v_{(t)}\left[\cos \alpha \cdot \cos \sigma_{0}+\sin \sigma \cdot \sin \sigma_{0}\right]
$$

Lo pocentiel des vitesses s'exprime aiors par:

$\dot{\phi}=2 b v_{(t)} \sum_{n=1,2}^{\infty} \frac{\operatorname{chn}\left(\alpha_{1}-\alpha_{1}\right)}{\operatorname{shn}\left(\alpha_{2}-\alpha_{1}\right)} \cdot e^{-n \alpha_{2}}\left(\cos \sigma_{0} \cos n ; \beta+\sin \sigma_{0} \sin n\right)$ 
Pour une vitesse de translation unite, on a sur le cercle $a_{a} a_{2}$ :

(b) $\alpha_{\alpha=a_{2}}=2 b \sum_{n=1,2}^{\infty} e^{-n \alpha_{2}} \cdot \operatorname{cth} n\left(a_{2}-\alpha_{1}\right)\left(\cos \sigma_{0} \cos n \beta+\sin \sigma_{0} \sin n \theta^{\prime}\right)$

$\left(\frac{\partial \beta}{\partial \alpha}\right)_{\alpha=\alpha_{2}}=2 b \sum_{n=1,2}^{\infty} n e^{-n \alpha_{2}}\left(\cos \sigma_{0} \cdot \cos n \beta+\sin \sigma_{0} \sin n \beta\right)$

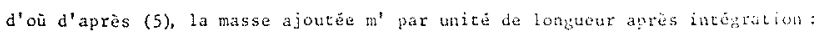

$$
m^{\prime}=4 p_{L} \pi b^{2} \sum_{n=1,2}^{\infty} n e^{-2 n a_{2}} \operatorname{cth} n\left(a_{2}-\alpha_{1}\right)
$$

La fígure 4 met en évidence, d'une part $1^{1}{ }^{\prime}{ }^{\prime}$ fifluence du confinement dû à l'enceinte cylindrique extérieure, et d'autre part l'effet de l'excentrment de la coque intérieure.

\subsection{Coque déformable}

Envisageons à titre drexemple le cas d'une coque respirant suivant le mode $\cos 2\left(\sigma-\sigma_{0}\right) ;$ les autres modes peuvent s'anaiyser de facon tout-à-fait similaire.

Dans ces conditions ha coque $\alpha_{2}$ est sounise is un mouveatent du type te? que $u \alpha_{2}=v(r) \cos 2\left(0-\sigma_{0}\right)$

Le potentiel des vitesses s'écrit :

$\phi=2 b \sum_{n=1,2}^{\infty} e^{-n \alpha_{2}}\left(n \operatorname{sh} \alpha_{2}-\operatorname{ch} \alpha_{2}\right) \frac{\operatorname{ch} n\left(\alpha-\alpha_{1}\right)}{\operatorname{shn}\left(\alpha_{2}-\alpha_{1}\right)}\left(\cos 2 \sigma_{0} \cos n i s+\sin 2 \sigma_{0} \sin n\right)$

L'intégration de la relation (5) conduit à la masse ajoutée par unité de longueur de coque :

$m^{\prime}=4 p_{1} \pi b^{2} \sum_{n=1,2}^{\infty} n\left(n \operatorname{sh} \alpha_{2}-\operatorname{cin} \alpha_{2}\right)^{2} e^{-2 n \alpha_{2}} \operatorname{cth} \pi\left(\alpha_{2}-\alpha_{1}\right)$

\subsection{Influence de la vitesse du fluide}

Considërons une coque placếe dans un écoulement perpendiculaire à l'axe de 1 a caque.

On désigne par :

$$
\begin{aligned}
& \text { a,e respectivement le rayon et l'épaisseur de la coque et posons : } \\
& k^{2}=12\left(\frac{a}{e}\right)^{2} \\
& w, v \text { déplacements radial et tangentiel de la coque, notations } \\
& \text { adimensionnelles } \mathrm{W}=\frac{\mathrm{w}}{\mathrm{a}} ; \mathrm{V}=\frac{\mathrm{v}}{\mathrm{a}} \\
& \text { 6 angle aziratal repérant un point de la coque } \\
& \dot{\forall}, \dot{v} \text { dórivées de } \boldsymbol{w} \text { et } y \text { par rapport au teraps } \\
& w^{\prime} \text { et v' dérivêes de wet } v \text { par rapport a } \theta
\end{aligned}
$$

Envisageons les nouvements dela paroi symécriques par rapport à 2 'axe $\theta=0$. Le déplacenent nornal de la coque peut se définir par le développenent :

$$
w=e^{\beta t} \sum_{n=1}^{\infty} w_{n} \cos n \sigma(1) \text { avec } 3 \text { amplitude complexe de la vibration }
$$

La vitesse radiale en un point de la coque s'exprime par :

$$
\dot{w}=B e^{B C} \sum_{n=1}^{\infty} w_{n} \cos n \theta
$$

Le fluide applique sur la paroi de la coque un pression extêrieure $p$ (variable adimensionnelle $P=\frac{\left(1-v^{2}\right) \text { a } p}{\text { Ee }}$; les charges extérieures par unicé de surface appliquées dans les conditions nornale at tangentielle sont donc égales à :

$$
\begin{aligned}
& p_{n}=-e \rho \ddot{w}-p=-e \rho \beta^{2} w-p \\
& p_{t}=-e \rho \ddot{v}=-e \rho \beta^{2} v
\end{aligned}
$$

Ecoulement induit par les vibrations :

$$
\text { Il dërive du potentiel } \infty=-a^{2} \sec \sum_{n=1}^{\infty} \frac{n_{n} a^{n}}{n r^{n}} \cos n \theta
$$

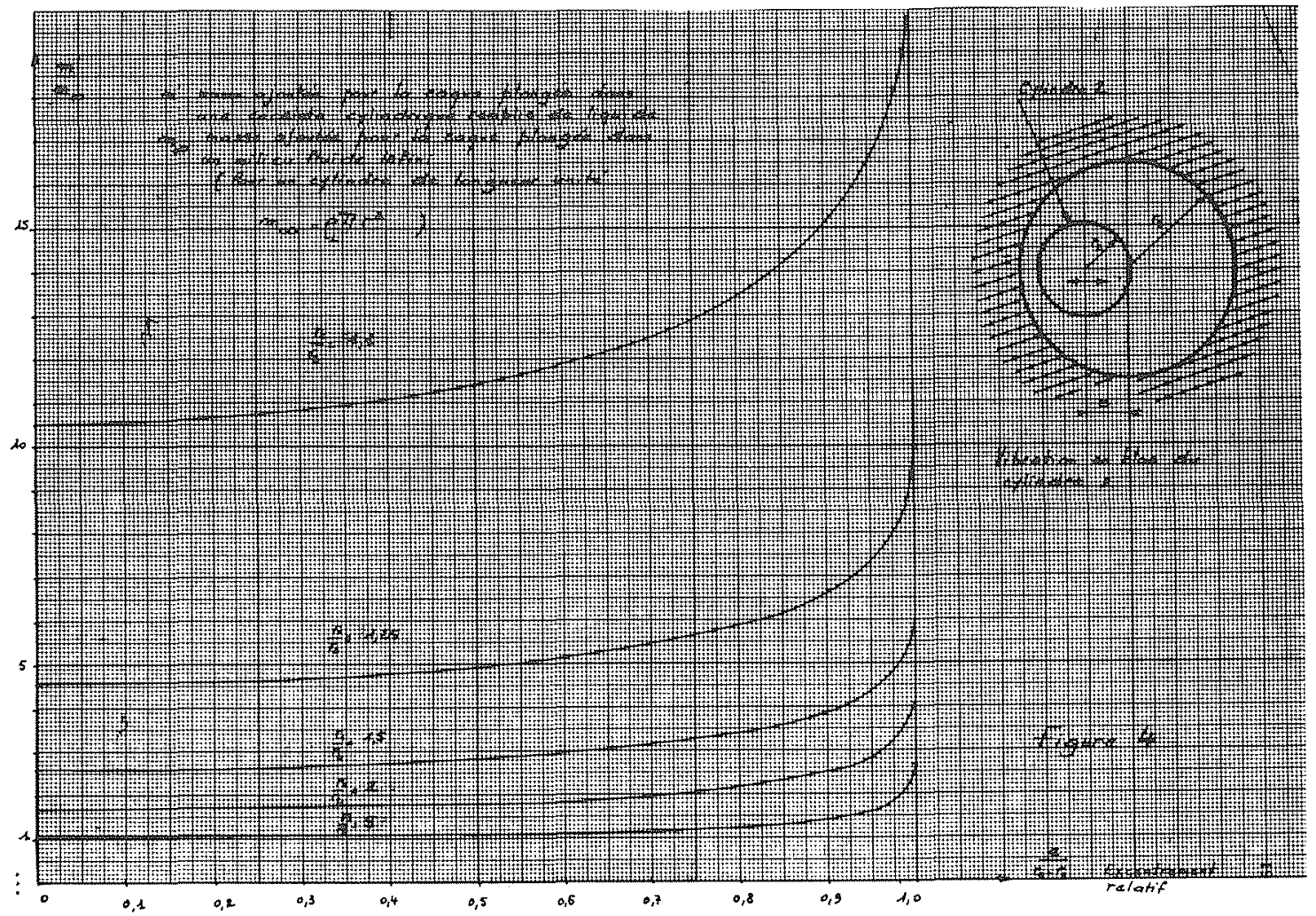

Figure 4 
Le champ des vitesses défini par ce potentiel satisfait bien aux conditions aux limites:

- il s'annule à l'infini,

- sur la paroi, la vitesse normale $\dot{h}^{\prime}$ est égale à la relation (2)

- Ecoulement extérieur dans lequel baigne la coque :

Nous supposons qu'en 1'absence de vibration cet êcoulement dêrive du potentiel

$$
\phi_{E}=-\frac{a v_{0}}{2}\left(\frac{a}{\tau}+\frac{5}{a}\right) \cos \theta
$$

qui donne, le long de la coque une vitesse tangentielle

$$
\dot{v}_{E}=v_{0} \sin \theta
$$

Dans la suite, les effets stationnaires ne seront pas considérés.

Le thếorème de Bernouilli linêarisê s'écrit :

$$
\frac{p}{p_{L}}=-\frac{\partial \phi}{\partial t}-\dot{v} \dot{v}_{E}-\dot{v} \dot{w}_{E}
$$

La vitesse radiale $\dot{\omega}_{E}$ correspondant à l'écoulement de base âtant nulle, la pression sur la coque est donnĕe par :

$$
\frac{P}{P_{L}}=-\left(\frac{\partial \Phi}{\partial t}\right)_{T=a}-\dot{V} \quad \dot{v}_{E}
$$

avec :

$$
\left[\frac{\partial \phi}{\delta t}\right]_{I=a}=-a^{2} \beta^{2} e^{\beta t} \sum_{n=1}^{\infty} \frac{W_{n}}{n} \cos n \theta
$$

$$
\begin{aligned}
& \text { et }:=\left(\frac{1}{r} \cdot \frac{\partial \phi}{\partial \theta}\right]_{r=a} \Rightarrow a \beta e^{\beta t} \sum_{n=1}^{\infty} w_{n} \sin n \theta \\
& \dot{v} \dot{v}_{E} a_{a} \beta v_{0} e^{\beta t} \sum_{n=1}^{\infty} w_{n} \sin n \theta \sin \theta \\
& \text { or } \sin n \theta \cdot \sin \theta=\frac{1}{2}\{\cos (n-1) \theta-\cos (n+1) \theta\}
\end{aligned}
$$

d'où :

$$
\dot{v} v_{E}=\frac{a \beta v_{0}}{2} e^{B t}\left(\sum_{n=1}^{\infty}\left(w_{n+1}-w_{n-1}\right) \cos n \theta+w_{1}\right)
$$

en posant : $\quad W_{0} * 0$

la pression au droit de la coque est donnée par :

$$
\frac{P}{Q_{L}}=a^{z B^{2}} e^{\beta t} \sum_{n=1}^{\infty} \frac{W_{n}}{n} \cos n \theta-\frac{a B}{2} V_{0} e^{B C}\left(W_{1}+\sum_{n=1}^{\infty}\left(W_{n+1}-W_{n-1}\right) \cos n \theta\right)
$$

soit en posant : $\quad \gamma^{2}=\frac{Q\left(1-v^{2}\right) a^{2} B^{2}}{E}$

$$
\begin{aligned}
& K=\frac{\rho_{L^{a}}}{\rho^{e}} ; \quad C^{2}=\frac{E}{\rho\left(1-v^{2}\right)} \\
& \alpha=\frac{K v_{0}}{2 C}
\end{aligned}
$$

$P=K \gamma^{2} \sum_{n=1}^{\infty} \frac{w_{n}}{n} \cos n \theta-\alpha \gamma\left(\omega_{1}+\sum_{n=1}^{\infty}+\sum_{n=1}^{\infty}\left(\omega_{n+1}-\omega_{n-1}\right) \cos n \theta\right)$

soit :

$$
P^{\prime}=K \gamma^{2} \sum_{n=1}^{\infty} w_{n} \sin n \theta+\alpha Y \sum_{n=1}^{\infty} n\left(w_{n+1}-w_{n-1}\right) \sin n \theta
$$

Si on désire étudier les vibrations de flexion de la coque cylindrique, les équations qui représentent les déformations correspondantes s'écrivent:

$(S)\left\{\begin{array}{l}v^{\prime \prime}=-a y-\frac{\left(1-v^{3}\right)}{E e} a^{2} p_{t}-\frac{a\left(1-v^{2}\right)}{E e} q \\ y^{\prime \prime}=-y-12\left(1-v^{2}\right) a^{2} q-\frac{\left(1-v^{2}\right)}{E e} q-a \frac{\left(1-v^{3}\right)}{E e} p t \\ q^{\prime \prime}=-q-a p_{i}-a p_{n}^{\prime}\end{array}\right.$

ou q représente l'effort de cisaillement par unité de longueur appliqué sur la lisière de l'élément considéré (variable adimensionnelle $\left.Q=\frac{\left(1-v^{2}\right)}{\operatorname{Ee}} q\right)$

$y=\frac{w^{\prime}}{a}$ dêformation angulaire de la coque au point considêré

Le déplacement en bloc n'étant pas considéré, nous considèrerons dans la suite $W_{1}=0$

Pour les mouvements symêtriques par rapport à 1 'axe $=0$ on doit avoir:

$$
y=V=Q=0 \quad \text { pour } \theta=0 \text { et } \theta=n
$$

Ces fonctions peuvent done sẹ reprêsenter par :

$$
\begin{aligned}
& v=e^{\beta t} \sum_{n=1}^{\infty} v_{n} \operatorname{sinn} n ; Q=e^{\beta t} \sum_{n=1}^{\infty} Q_{n} \operatorname{sinn} n \\
& y=-e^{\beta t} \sum_{n=1}^{\infty} n w_{n} \operatorname{sinn~} \theta \quad \text { d'après (i) }
\end{aligned}
$$

avec d'après la condition (6) $w_{1}=V_{1}=Q_{1}=0$

Compte tenu des relations (3) et (4) le systême (5) s'êcrit donc :

$$
\begin{aligned}
& v^{\prime \prime}=-y+\gamma^{2} v-Q \\
& y^{\prime \prime}=-y-\left(k^{2}+1\right) Q+\gamma^{2} v \\
& Q^{\prime \prime}=\gamma^{2} y-Q+\gamma^{2} v+p^{\prime}
\end{aligned}
$$

soit par identification terme à terae pour $n \geqslant 2$ avec $H_{1}=0$

$$
\begin{aligned}
& \left(n^{2}+Y^{2}\right) v_{n}=Q_{n}-n W_{n} \\
& n\left(n^{2}-1\right) W_{n}=Y^{2} v_{n}-\left(k^{2}+1\right) Q_{n} \\
& \left(n^{2}-1\right) Q_{n}=-Y^{2} v_{n}+\gamma^{2}(K+n) w_{n}+\alpha Y_{n} W_{n}-1-\alpha Y_{n} W_{n}+1
\end{aligned}
$$

Systême qui s'êcrit encore :

$$
a_{n(n-1)} w_{n-1}+a_{n n} w_{n}+a_{n}(n+1) w_{n+1}=0
$$

où :

$$
\begin{aligned}
& a_{n n}=1+\frac{\left(n^{2}+1\right)\left(k^{2}+n^{2}\right)+n k\left(k^{2}+1\right)}{n^{2}\left(n^{2}-1\right)^{2}} \gamma^{2}+\frac{k^{2}(k+n)}{n^{3}\left(n^{2}-1\right)^{2}} \gamma^{4} \\
& a_{n(n-1)}=-a_{n(n+1)}=\frac{\alpha \gamma\left(k^{2}+1\right) n^{2}+k^{2} \alpha \gamma^{3}}{n^{2}\left(n^{2}-1\right)^{2}}
\end{aligned}
$$

Le dêterminant caractéristique s'écrit :

$$
\left|\begin{array}{llll}
a_{22} & a_{23} & \\
a_{32} & a_{33} & a_{34} & \\
& a_{43} & a_{44} & a_{45}
\end{array}\right|=0
$$




$$
\begin{aligned}
& \text { Les xacines de: } \\
& D_{33}=\frac{a_{22}=0}{a_{22} a_{33}-a_{32} a_{23}}=0 \\
& D_{44}=\frac{a_{44} D_{33}-a_{34} a_{43}}{a_{66}}=0 \text { etc... tendent vers les racines } \\
& \text { du déterminant }
\end{aligned}
$$

A titre d'exemple, considêtrons une coque en acier de rapport $\frac{a}{e}=10$

et baignée extérieurement par de l'eau dont la vicesse tangentielle maximale en parol atteint $100 \mathrm{~m} / \mathrm{s}$. L'étude précédente montre que cet écoulement rapport au cas oil le Eluide serait initialea propre repos.

Rappelons toutefois que si du point de vue des masses ajoutées, l'influence du mouvement du fluide peut être considérée conme faible, il peut engendrer une amplification des vibrations de la coque par suite de :

l'instabilité de l'écoulement lui-même (dêcollements, sillages, tourbillons de Benard Karman), la présence du fluide en mouvement qui constitue lui-même un apport continu d'ênergie.

BIBLIOGRAPHIE

Vibrations d'un panneau rectangulaire encastré, seule face flexible d'une enceinte paralléllépipédique remplie d'un Eluide incompressible.

$$
\text { S. CASACCI (S.F.M.) }
$$

Calcul bidimensionnel de la matrice masse ajoutée crêé par un fluide sêparant deux coques cylindriques circulaires d'axes parallêles décentrếs.

$$
\text { R.J. GIBERT (Note Technique C.E.A) }
$$

Masses ajoutées de coques cylindriques excentrêes baignant dans un fluide

$$
\text { S. CASACCI - I. EREMEEF (Rapport interne NEYRPIC) }
$$

\section{Discussion}

\section{Président : M. S. CASACCI}

M. le Président. - Je remercie M. CAILLot de cet exposé. Je pense nécessaire de préciser ce qu'il entend par conditions d'adhérence. En fait, en écoulement parfait, seule compte la vitesse normale à la paroi puisqu'il peut $\mathrm{y}$ avoir glissement du fluide le long de la paroi.

M. BINDER. - Ne pourrait-on pas considérer l'influence de la viscosité ? L'effet de la viscosité peut changer complètement l'écoulement potentiel.

M. le Président. - Il faut sérier les difficultés. Deux caractéristiques principales influent sur le comportement dynamique des structures:

- les modes et les fréquences propres en présence d'un milieu fluide,

- l'amortissement dû à la viscosité du fluide.

Les études analy tiques se rapportant à des cas relativement simples montrent que l'influence de la viscosité sur les modes et les fréquences propres reste pratiquement négligeable. L'hypothèse du fluide parfait et du mouvement à potentiel se justifie donc lorsqu'on s'intéresse aux risques de résonance.

L'évaluation de l'amortissement dû à la viscosité du fluide peut se calculer pour des cas simples en supposant des vibrations de très faible amplitude, ce qui permet de négliger les termes convectifs d'accélération. Malheureusement les valcurs do l'amortissement réduit calculé ne correspondent pas aux résultats de mesures effectuées sur des modèles ou des structures industrielles. En fait, l'amortissement est un phénomène complexe.

Pour revenir à la détermination des modes et des fréquences propres, les exemples traités par M. Caillot montrent que l'effet de confinement est primordial. L'influence de la vitesse ambiante du fluide reste faible sur les fréquences, sauf bien entendu si l'existence de ce mouvement provoque $d$ 'au tres phénomènes tels qu'instabilité hydro-élastique ou flambage de la structure.

M. GIBERT. - L'effet de la viscosité du fluide a un rôle essentiellement sur l'amortissement de la structure vibrante. Exemple : Deux coques cylindriques concentriques, l'une rigide (épaisseur grande) l'autre vibrante (épaisseur $\sim 0,5 \mathrm{~mm}$ ), séparées par une lame d'eau d'épaisseur $5 \mathrm{~mm}$ (diamètre des cylindres $\sim 200 \mathrm{~mm}$ ).

Un calcul analy tique bi-dimensionnel peut être effectué en supposant le fluide visqueux.

Une comparaison expérience-calcul en fluide visqueux ou non visqueux montre une bonne concordance en ce qui concerne les fréquences de résonance des premiers modes (les effets d'inertie du fluide, très importants dans ce cas, sont donc bien estimés par le calcul).

En ce qui concerne les amortissements modaux, ils sont de l'ordre de $10^{-2}$ (le calcul en fluide visqueux retrouve cet ordre de grandeur). L'effet de la viscosité est donc faible vis-à-vis de l'effet d'inertie. Il est cependant important de le considérer pour l'estimation des amortissements qui sont des paramètres essentiels de l'analyse vibratoire des structures.

Ce qui vient d'être dit précédemment ne s'applique pas à certaines configurations particulières où le confinement est extrême : par exemple, les paliers fluides des machines tournantes. Dans ce cas, l'effet de la viscosité est prépondérant.

M. SAGNER. - On peut évaluer l'ordre de grandeur de l'influence de la viscosité en considérant l'équation du fluide, linéarisée pour les petits mouvements. Elle a la forme d'une équation de la chaleur et montre que la perturbation due à la viscosité se fait sentir dans une couche limite d'épaisseur $\sqrt{\nu / \omega}$ alors que l'effet d'inertie correspond au champ de vitesse potentiel qui se fait sentir sur une distance de l'ordre des dimensions du corps. Si le nombre de Reynolds $\omega^{2} L / v$ est très grand, le rapport de ces deux grandeurs est très petit et l'effet de viscosité est négligeable.

M. BINDER. - Dans quelle mesure les effets de la viscosité modifient-ils les résultats obtenus pour le fluide parfait, notamment lorsqu'il y a décollement?

M. le Président. - Le comportement des structures plongées dans des écoulements fortement perturbés fera l'objet d'un premier examen dans l'exposé relatif à l'effet des détachements tourbillonnaires du sillage.

Les études présentées ici se rapportent à la détermination des modes et des fréquences des structures vibrant en présence d'un milieu fluide initialement au repos. Les amplitudes des vibrations sont supposées très faibles. Dans le cas d'un fluide en mouvement, le calcul montre sur des exemples simples que la vitesse ambiante du fluide n'a que peu d'influence si le phénomène reste stable. Nous n'avons pas traité le cas des instabilités hydroélastiques.

M. SAGNER, - Dans le seul cas où on a étudié l'influence d'un décollement (celui du cylindre circulaire) on constate que celui-ci n'apparaît que pour des grandes amplitudes de vibration. Il se traduit principalement par un terme d'amortissement élevé et une réduction modérée du terme d'inertie. Tout se passe comme si la force se déphasait par rapport à l'accélération en conservant sensiblement son module. 